\title{
Construction and Simulated Experiment of Comprehensive Evaluation Model for Information Policy Scheme
}

\author{
Mingwei Sun ${ }^{1}$, Li Zhang ${ }^{2, *}$ \\ 'School of Computer, Tonghua Normal University, Tonghua, 134002, China \\ ${ }^{2}$ School of Physics, Tonghua Normal University, Tonghua, 134002, China \\ *dbcy9999@qq.com
}

\begin{abstract}
Key words: Information Policy Plan, Evaluation Indicates System, Fuzzy Comprehensive Evaluation Method.
\end{abstract}

\begin{abstract}
The scientific evaluation of information policy plan is the basis of the implementation of information policy. This paper proposes the comprehensive evaluation indicates system of the information policy scheme, constructs the evaluation model by using fuzzy comprehensive evaluation method, and has confirmed the model's feasibility and practicability by simulated experiment.
\end{abstract}

\section{Introduction}

China's information policy research began in the late 1970s, due to the important position and indispensable role of information policy in the national information construction[1], the problem of studying the information policy making plan is becoming more and more prominent. How to evaluate the information policy scheme and make rational and effective use of it to guide the implementation of information policy has become an important subject of quantitative and scientific research on information policy[2].

\section{Evaluation of information policy scheme and fuzzy comprehensive evaluation}

The Information policy scheme is the basic premise of all information policy implementation, and also an important guarantee for the scientific operation of information policy. The quality of the scheme is not simply evaluated by a single factor, but by a variety of factors[3], which requires a comprehensive evaluation method, and the evaluation results are often very good, generally good, bad with fuzzy color evaluation[4], Because of the above reasons, this paper uses the fuzzy comprehensive evaluation method to predict the information policy scheme[5]. It not only solves the complex problem of multi-factor influence, but also meets the fuzziness requirement of comment.

\section{Constructing Fuzzy Comprehensive Evaluation Model of Information Policy Scheme}

3.1 Determination of the comprehensive evaluation index system (factor set) and evaluation set of information policy programmes

The author puts forward an index system which is composed of basic indexes according to the principle of constructing the evaluation index system of information policy scheme[6]. The factor set is $\mathrm{U}=\{\mathrm{u} 1, \mathrm{u} 2, \mathrm{u} 3 \ldots \mathrm{u} 10\}$, and the commentary is $\mathrm{V}=\{\mathrm{v} 1, \mathrm{v} 2, \mathrm{v} 3, \mathrm{v} 4, \mathrm{v} 5\}$. For different factors and the value of information, the 5 level commentary focused on different meanings[7]. The specific meaning of the comment set corresponding to different factors is shown in Table 1. 
Table 1. comprehensive evaluation indicators of information policy options (factors) and corresponding Reviews

\begin{tabular}{cc}
\hline Factor set U & Comment set V \\
\hline Clarity of objectives u1 & [Very clear, clear, relatively clear, fair, ambiguous] \\
\hline Innovativeness u2 & [Very novel, novel, relatively novel, fair, old] \\
\hline Timeliness u3 & [Very strong, strong, relatively strong, fair, weak] \\
\hline Scientific u4 & [Very strong, strong, relatively strong, fair, weak] \\
\hline Normative u5 & [Very standardized, standardized, relatively standardized, \\
fair, non-standardized]
\end{tabular}

\section{2 determination of the factor weighting coefficient Wi for judging information policy schemes}

In each basic evaluation index, the degree of reflection and influence of each index on the information policy scheme is different, that is, the weight is different, so it is necessary to distribute the weight of each index[8]. Practice has proved that AHP is especially suitable for dealing with complex problems which are difficult to complete quantitative analysis. Therefore, this paper intends to use AHP analytic hierarchy process to determine the weights of each index.

\subsubsection{Comparative judgement and determination of comparative order of importance of factors}

The psychological experiments show that most people have the ability to distinguish between the same attributes of different things in the range of 1-9. Therefore, the 1-9 scale method proposed by American Professor SAATY is used to reflect the judgment ability of most people[9]. According to the scale method, this paper compares the indexes of each layer, constructs a judgment matrix, and assigns the value according to the scale of 1-9.

\subsubsection{Establishes judgement matrix and determines relative weight.}

According to the rules of the scale law, experts determine the size of the evaluation factors U1 U10 of the model to play a role in the evaluation of the target, two comparisons, get the judgment matrix. Assuming that the relative weight of the evaluation factor $\mathrm{u} 1-\mathrm{u} 10$ of the model is Wi $(i=1,2,3 \ldots, 10)$, the relative weight $\mathrm{Wi}$, the consistency index $\mathrm{CI}$, the maximum characteristic root and the random consistency ratio $\mathrm{CR}$ of each factor can be calculated by MATLAB 7.0 simulation software.

Table 2 the judgement matrix and relative weight table of factor set $\mathrm{U}$

\begin{tabular}{llllllllllll}
\hline $\mathrm{U}$ & $\mathrm{u} 1$ & $\mathrm{u} 2$ & $\mathrm{u} 3$ & $\mathrm{u} 4$ & $\mathrm{u} 5$ & $\mathrm{u} 6$ & $\mathrm{u} 7$ & $\mathrm{u} 8$ & $\mathrm{u} 9$ & $\mathrm{u} 10$ & $\mathrm{Wi}$ \\
\hline $\mathrm{u} 1$ & 1 & 2 & 3 & 2 & 5 & 3 & 2 & 3 & 2 & 3 & 0.194 \\
\hline $\mathrm{u} 2$ & $1 / 2$ & 1 & 3 & 2 & 3 & 3 & 2 & 3 & 2 & 2 & 0.157 \\
\hline $\mathrm{u} 3$ & $1 / 3$ & $1 / 3$ & 1 & $1 / 3$ & 3 & $1 / 2$ & $1 / 3$ & $1 / 3$ & $1 / 4$ & $1 / 5$ & 0.037 \\
\hline $\mathrm{u} 4$ & $1 / 2$ & $1 / 2$ & 3 & 1 & 3 & 2 & 2 & 2 & 2 & 3 & 0.136 \\
\hline $\mathrm{u} 5$ & $1 / 5$ & $1 / 3$ & $1 / 3$ & $1 / 3$ & 1 & $1 / 3$ & $1 / 5$ & $1 / 3$ & $1 / 4$ & $1 / 5$ & 0.026 \\
\hline $\mathrm{u} 6$ & $1 / 3$ & $1 / 3$ & 2 & $1 / 2$ & 3 & 1 & $1 / 4$ & $1 / 4$ & $1 / 5$ & $1 / 6$ & 0.042 \\
\hline $\mathrm{u} 7$ & $1 / 2$ & $1 / 2$ & 3 & $1 / 2$ & 5 & 4 & 1 & 2 & 3 & 2 & 0.128 \\
\hline $\mathrm{u} 8$ & $1 / 3$ & $1 / 3$ & 3 & $1 / 2$ & 3 & 4 & $1 / 2$ & 1 & 2 & $1 / 2$ & 0.082 \\
\hline $\mathrm{u} 9$ & $1 / 2$ & $1 / 2$ & 4 & $1 / 2$ & 4 & 5 & $1 / 3$ & $1 / 2$ & 1 & $1 / 2$ & 0.084 \\
\hline $\mathrm{u} 10$ & $1 / 3$ & $1 / 2$ & 5 & $1 / 3$ & 5 & 6 & $1 / 2$ & 2 & 2 & 1 & 0.114 \\
\hline
\end{tabular}

$\lambda_{\max }=11.103$, 


\subsection{Determination of Fuzzy Comprehensive Evaluation matrix for information policy schemes.}

Each factor in the factor set $\mathrm{U}$ is evaluated, and the fuzzy mapping f from $\mathrm{U}$ to $\mathrm{V}$ is obtained, from which the fuzzy relation $\mathrm{Ri} \in \mathrm{F}(\mathrm{Ui} \times \mathrm{V}), \mathrm{i}=1,2$. The members of the expert group evaluate the factors of the information policy scheme according to the evaluation set, and construct the evaluation vector and fuzzy matrix of each factor from the evaluation results. For example, the evaluation vector of the first factor of the set $U$ is (ri1, ri2, ri3, ri4,ri5) ( $i=1,2, \cdots 10)$ The fuzzy evaluation matrix of the corresponding set of factors $U$ is as follows:

$$
\underset{\sim}{R}=\left|\begin{array}{cccc}
\mathrm{r} 11 & \mathrm{r} 12 & \ldots & \mathrm{r} 15 \\
\mathrm{r} 21 & \mathrm{r} 22 & \ldots & \mathrm{r} 25 \\
\vdots & \vdots & \vdots & \vdots \\
\text { ri1 } & \text { ri2 } & \ldots & \text { ri5 } \\
r_{i j} & \text { experts rated as J level }
\end{array}\right|
$$

Among $r_{i j}=$ experts rated as $\mathrm{J}$ level / the total number of experts $(\mathrm{i}=1,2 \ldots 10, \mathrm{j}=1,2, \ldots 5)$, which can be interpreted as the subordinate factor of the I level of comment $J$.

\subsection{The result of Fuzzy Comprehensive Evaluation of calculating information policy scheme}

The evaluation vector of information policy plan is $\mathrm{V}=\mathrm{W} \cdot \mathrm{R}=\left(v_{c 1}, v_{c 2} \ldots v_{c 5}\right), v_{c i}$ $(\mathrm{i}=1,2, \cdots, 5)$ membership degree of the information policy scheme to the I level comment. The final comment level is (the best, better, good, fair, bad). According to the principle of maximum subordinate degree, the comment on the evaluated information policy is $v_{c \max }=\max v_{c i} \quad(1 \leqslant \mathrm{i} \leqslant 5)$.

\section{Model simulation experiment}

Now 10 experts evaluate a certain information policy scheme according to the model set up in this paper. Firstly, the evaluation level of each influencing factor is determined, Secondly, the fuzzy evaluation matrix of the factor set $U$ of the scheme is determined as follows:

$$
\underset{\sim}{R}=\left|\begin{array}{ccccc}
0.1 & 0.9 & 0.1 & 0 & 0 \\
0 & 0.3 & 0.8 & 0 & 0 \\
\vdots & \vdots & \vdots & \vdots & \vdots \\
0.1 & 0.3 & 0.4 & 0 & 0
\end{array}\right|
$$

Finally, the evaluation vector of the information policy scheme is $\mathrm{V}=\mathrm{W} \cdot \mathrm{R}=(0.1,0.195,0.157$, $0.037,0.026)$ and normalized $\mathrm{V}=(0.18,0.40,0.29,0.08,0.05)$. The results showed that $18 \%$ thought the scheme was the best, $40 \%$ thought it was better, $29 \%$ thought it was good, $8 \%$ thought it was fair, and $5 \%$ thought it was bad. According to the principle of maximum membership $v_{c \max }=0.4$ can be finally determined. That is, the information policy scheme is a good and can be implemented.

\section{Summary}

In this paper, the scheme of information policy is discussed and a fuzzy comprehensive evaluation model of information policy is established to solve the problem of whether the scheme of information policy is reasonable and effective, so as to ensure the correct implementation of information policy and achieve the desired results.

\section{Acknowledgement}

This research was financially supported by Research Foundation of Education Bureau of Jilin Province (Grant No.JJKH20180860KJ). 


\section{References}

[1] Zhao Dapeng, Zhang Ruixin. Analysis of Information Policy Making Strategies in China. Modern Intelligence, 2009 (4): 20-22.

[2] Ma Haiqun, Qi Zengmei. Analysis of the Disciplined Process and Basic Problems of Information Policy Research. Journal of Information Science, 2007 (1): 42-49.

[3] Ma Haiqun, Zhang Dandan. Research on the Operating Mechanism of Information Policy System. Library Forum, 2005 (12): 262-266.

[4] Qin Shoukang. Comprehensive Evaluation Principle and Application. Beijing: Electronic Industry Publishing House, 2003.

[5] Li Shiyong. Engineering Fuzzy Mathematics and Its Application. Harbin: Harbin University of Technology Press, 2004:98-103.

[6] Xia Guoen, Shao Peiji. Comprehensive Evaluation of Business Intelligence System Based on Support Vector Machine. Computer Application Research, 2009 (5): 1789-1790.

[7] Song Jianfeng, Yuan Ruhua. Construction of Policy Evaluation Index System. Statistics and Decision-making, 2006 (11): 63-64.

[8] Qi Liangqun. Design of policy management system for high-tech industries. China Soft Science, 2005 (3): 139-144.

[9] Yang Sheng. Using AHP to construct information service quality evaluation system. Information Journal, 2005 (11): 22-24. 